\title{
CULTIVO MASIVO DE ROTIFEROS N ESTANQUES DE TIERRA
}

Gilberto A scon Dionicio (*)

\section{RESUMEN}

La presente investigación ha tenido por objeto dar a conocer un método para obtener la producción masiva de rotíferos. En el experimento se utilizaron 8 estanques de tierra de $4 \mathrm{~m} 2$

En base del análisis cualitativo se determinó la presencia de protozoarios, rotíferos y cladóceros. El tratamiento más significativo fue el 12 , que consideró $1.5 \mathrm{Kg}$. de galhnaza/m2 + $30 \mathrm{~g}$. de superfosfato/m2 con una producción masiva de rotíferos del género Brachionus de $78 x$

Palabras claves Rotíferos, 'gallinaza', superfosfato, cultivo de larvas y alevinos Brachionus.

\section{ABST RACT}

This paper describes a method in order to obtain the masive production of Rotifera. It was used 8 gruound ponds of $4 \mathrm{~m}^{2}$.

It was determined trough of ten qualitative analysis the presence of Protozoa, Rotifera and Cladocera. Trought of the treatment T2. It was obtain the massive production production of Rotifera, with a maxim concentration of $18 \times 103 \mathrm{ml} / \mathrm{l}$ and 78 x 10-3 mi/l, respectively.

\section{INTRODUCCION}

Se considera que las mayores dificultades que se presentan para la producción masiva de alevinos mediante la reproducción inducida de «gamitana» Colossoma macropomum y «paco» Piaractus brachypomus, consiste en mantener la supervivencia en la fase larva-alevino. U no de los factores predominantes que más gravita es la falta de alimento vivo en calidad y cantidad suficiente.

Teniendo en cuanto los resultados positivos de los ensayos preliminares sobre producción de alimento vivo realizados por Ascón y A renas (1986), se ha estimado conveniente orientar la presente investigación a la producción masiva de rotíferos, ya que se considera que este grupo de microcrustáceos constituye un excelente alimento a utilizar en los estadios larvales de los peces, crustáceos y moluscos. Entre los trabajos

Instituto de Investigaciones de la A mazonia Peruana - IIAP Centro Regional de Investigación San M artín - 
similares realizados destacan los efectuados por Elsie lech (1982), Hirata y Non (1\% 7), Hidaka (1973), M andy et al (1977) y Rodríguez V alderrama, A . (1983).

\section{MATERIAL Y METODOS}

El experimento fue realizado en 8 estanques de tierra de $4 \mathrm{~m} 2(2 \times 2 \times 0.5)$, del 8 al 19 de mayo de 1987, en la Estación de Pesquería de A huashiyacu, provincia y región de San M artín.

Los estanques fueron encalados a razón de $150 \mathrm{~g} /$ estanque, luego se le llenó con agua hasta un nivel de $0.25 \mathrm{~m}$ de altura, sin renovación de agua; dichos estanques fueron fertilizados con estiercol de gallina (ponedoras) y superfosfato triple, con $29.83 \%$ de N, $17.54 \%$ de PO4 $52.63 \%$ de K. Se aplicó el diseño completamente al azar, con 3 tratamientos, de 2 y 3 repeticiones, tal como se indica a continuación:

$\mathrm{Ti}=1.0 \mathrm{Kg} \cdot$ (gallinaza) $/ \mathrm{m} 2+30 \mathrm{~g}$. (superfosfato)! $\mathrm{m} 2 \times 2$ réplicas.

$12=1.5 \mathrm{~kg}$. (gallinaza) $/ \mathrm{m} 2+30 \mathrm{~g}$. (Superfosfato) $! \mathrm{m} 2 \times 3$ réplicas.

$13=2.0 \mathrm{Kg}$. (gallinaza) $/ \mathrm{m} 2+30 \mathrm{~g}$. (superfosfato) $/ \mathrm{m} 2 \times 3$ réplicas

DISTRIBUCION AL AZAR

\begin{tabular}{|l|cccccccc|}
\hline $\mathrm{N}^{2}$ DE ESTANQUE & 1 & 2 & 3 & 4 & 5 & 6 & 7 & 8 \\
\hline Tratamientos & $\mathrm{T}_{3}$ & $\mathrm{~T}_{1}$ & $\mathrm{~T}_{2}$ & $\mathrm{~T}_{1}$ & $\mathrm{~T}_{2}$ & $\mathrm{~T}_{2}$ & $\mathrm{~T}_{3}$ & $\mathrm{~T}_{3}$ \\
\hline Kg. (gallinaza)/Est. & 8 & 4 & 6 & 4 & 6 & 6 & 8 & 8 \\
\hline g. (superf.)/ Est. & 120 & 120 & 120 & 120 & 120 & 120 & 120 & 120 \\
\hline
\end{tabular}

Con el fin de realizar los análisis cuali y cuantitativos de zooplancton, se hicieron muestreos diarios a los 9 horas, a partir del segundo día de iniciado el experimento, utilizando una red para plancton de $60 \mathrm{u}$ de abertura de malla.

EI análisis cualitativo se realizó utilizando un microscopio binocular marca NIKON x 10 $X 40 x$ y las claves para rotíferos de Waler Kaste (1972) y Olivier Raúl (1\%5).

Para el análisis cuantitativo se aplicó el método volumétrico descrito por Tresierra, A. et al (1981): que consiste en centrifugar el zooplancton concentrado, luego se lee el volumen ocupado por el zooplancton en el tubo de centrifugación; para el cálculo se aplicó la fórmula siguiente:

Volumen de $=\frac{\text { volumen de zooplancton centrifugaración (ml) }}{\text { Zooplancton volumen de agua filtrada (1) }}$ 
Los registros de Temperatura y pH se hicieron a lo largo de todo el proceso, utilizando un termómetro demercurio graduado de $0^{\circ}-50^{\circ} \mathrm{C}$ y con una cinta indicadora de $\mathrm{pH}$, que varía de 0-14.

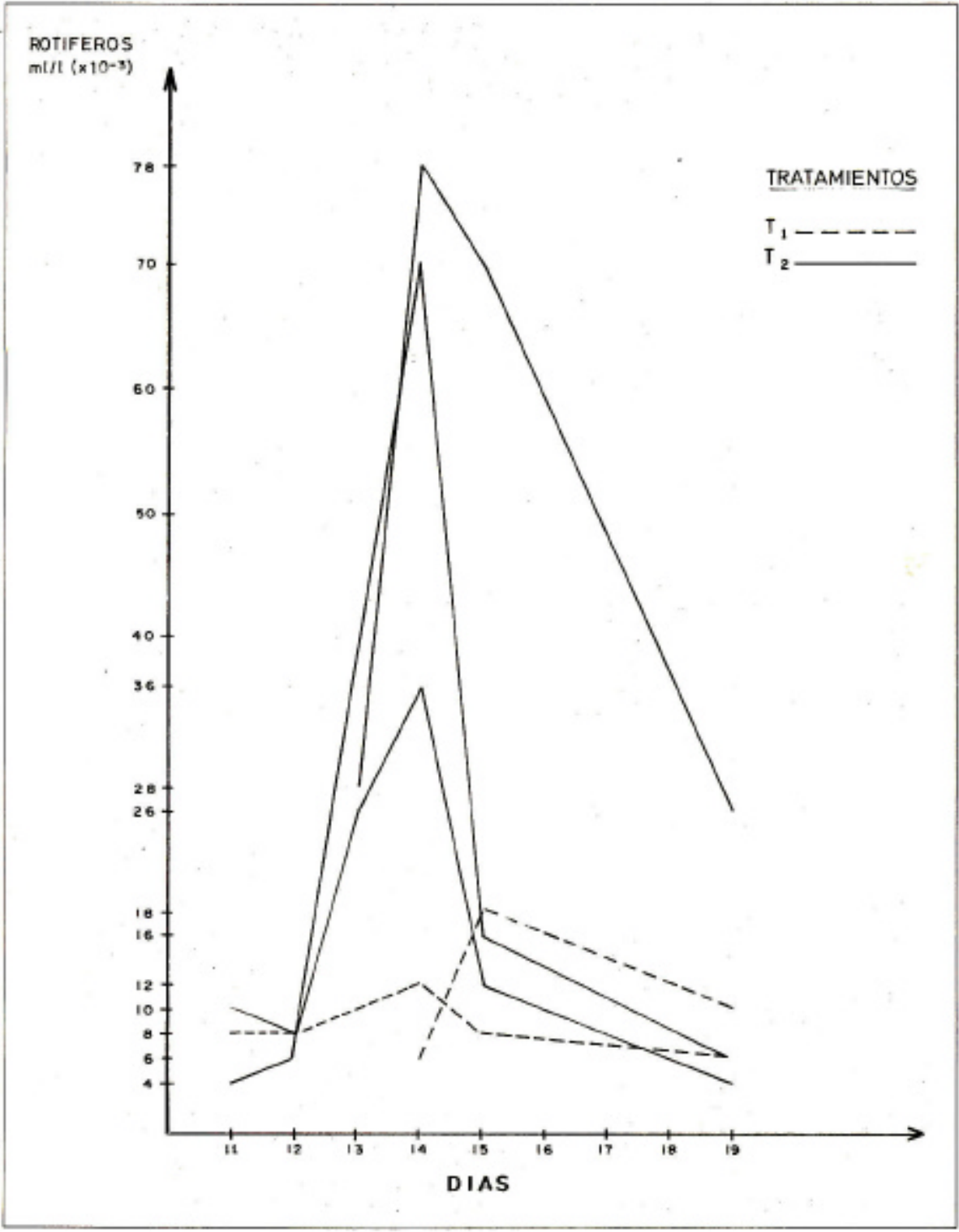

FIGURA 1: CANTIDAD DE ROTIFEROS DEL GENERO BRACHIONUS EN $\mathrm{ml} / /\left(\times 10^{-3}\right)$ EN ESTANQUES DE TIERRA DE: $4 \mathrm{~m}^{2}$ 


\section{RESULTADOS Y DISCUSION}

En el presente ensayo se pudo apreciar que el zooplancton apareció después las 5horas de iniciado el experimento, con la presencia de algunos protozoos. Los rotíferos aparecieron al segundo día, lo mismo que las larvas de insectos. El rotífero predominante y que se tomó masivo perteneció al género Brachionus; los resultados del análisis cualitativo se pueden apreciar en las Tablas 1 y 2.

Los resultados del análisis cuantitativo se pueden ver en la Tabla 3 y en la Figura 1, donde se aprecia que el tratamiento T2 dio mejores resultados que el tratamiento $\mathrm{Ti}$, con una producción masiva de $78 \times 10^{-3 \mathrm{ml} / 1}$ de rotíferos del género Brachionus.

Si comparamos los resultados de los experimentos realizados en estanques de tierra con los obtenidos en estanques de cemento, se puede apreciar que el ensayo realizado en estanques de tierra dio mejores resultados.

Los registros de T0 y pH oscilaron entre 27.5 a $29.5^{\circ} \mathrm{C}$ y de 6-7, respectivamente.

TABLA 1: $\quad$ Resultados del análisis cualitativo en estanques de tierra (experimento $\mathrm{N} 03$ )

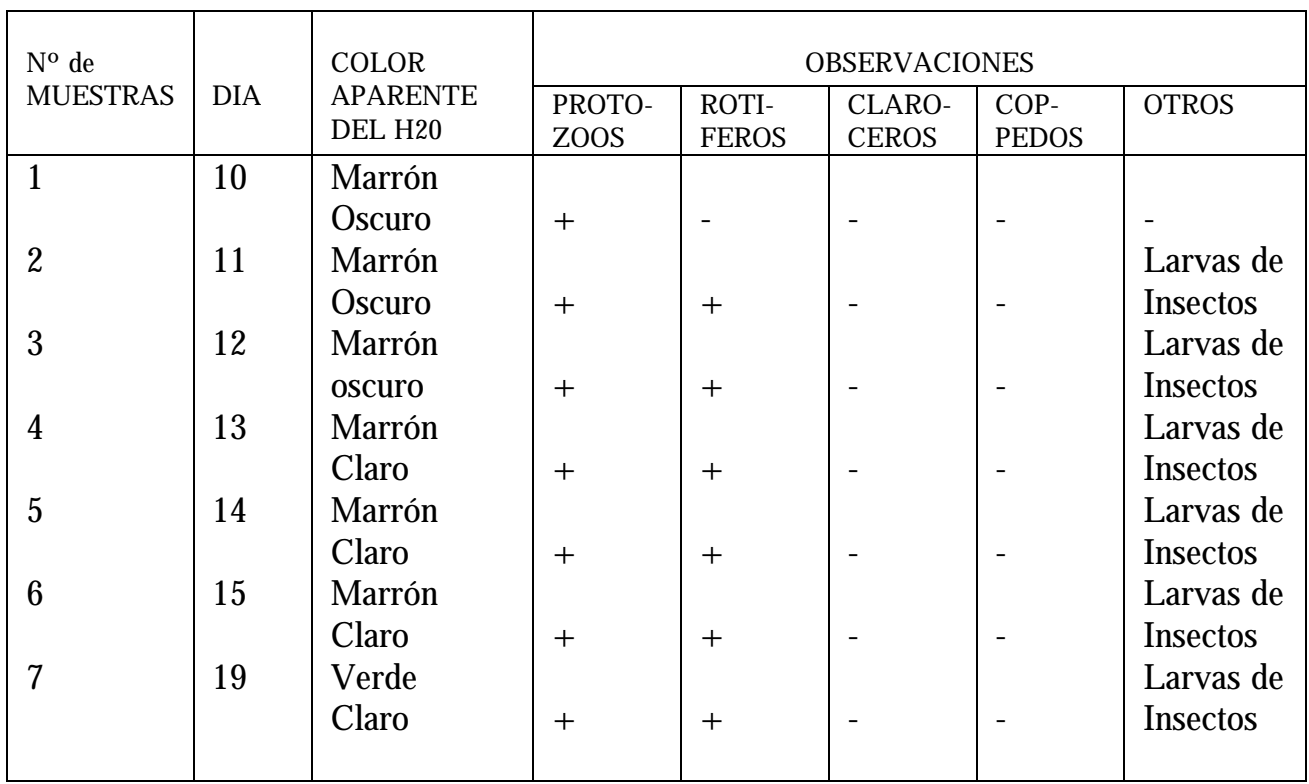

(+ ) Presencia (-) A usencia 
TABLA 2: Divisiones y géneros encontrados en el análisis cualitativo de zooplancton y su abundancia (Experimento $\mathrm{N} N 3$ )

\begin{tabular}{|l|ll|}
\hline \multicolumn{1}{|c|}{ DIVISION } & \multicolumn{1}{|c|}{ GENEROS } & VALORES DE \\
& & ABUNDANCIA \\
\hline Protozos & Paramecium & + \\
& Borticella & ++ \\
& Volvox & ++ \\
& Euglena & ++ \\
Rotifera & A splachna & + \\
& Phylodina & ++ \\
Cladócera & Brachionus & +++ \\
& Moina & + \\
\hline
\end{tabular}

$(+)$ = Escaso; $(++)$ = Poco abundante; $(+++)=$ M uy A bundante.

TABLA 3: Candidato de rotífero del género Brachionus por $\left(\mathrm{ml} / \mid \mathrm{x} 10^{-3}\right)$ en estanques de Tierra

\begin{tabular}{|c|c|c|c|c|c|c|c|c|}
\hline \multirow{3}{*}{$\begin{array}{l}\text { INICIO } \\
\text { No DE } \\
\text { ESTAN- } \\
\text { QUE }\end{array}$} & \multicolumn{8}{|c|}{$09-05-87$} \\
\hline & \multirow{2}{*}{$\begin{array}{l}\text { TRAT } \\
\text { A- } \\
\text { MIEN- } \\
\text { TOS } \\
\end{array}$} & \multicolumn{4}{|c|}{ DIA } & \multicolumn{3}{|c|}{ MUESTRADOS } \\
\hline & & 10 & 11 & 12 & 13 & 14 & 15 & 19 \\
\hline 1 & $T^{3}$ & - & - & - & & & & \\
\hline 2 & $T^{1}$ & - & - & _ & - & $6 \times 10^{-3}$ & $18 \times 10^{-3}$ & $10 \times 10^{-3}$ \\
\hline 3 & $T^{2}$ & - & $4 \times 10^{-3}$ & $6 \times 10^{-3}$ & - & $70 \times 10^{-3}$ & $16 \times 10^{-3}$ & $6 \times 10^{-3}$ \\
\hline 4 & $T^{1}$ & - & $8 \times 10^{-3}$ & $8 \times 10^{-3}$ & & $12 \times 10^{-3}$ & $8 \times 10^{-3}$ & $6 \times 10^{-3}$ \\
\hline 5 & $T^{2}$ & - & $10 \times 10^{-}$ & $8 \times 10^{-3}$ & $26 \times 10^{-3}$ & $36 \times 10^{-3}$ & $12 \times 10^{-3}$ & $4 \times 10^{-3}$ \\
\hline 6 & $T^{2}$ & - & 3 & - & $28 \times 10^{-3}$ & $78 \times 10^{-3}$ & $70 \times 10^{-3}$ & $26 \times 10^{-3}$ \\
\hline 7 & $T^{3}$ & . & - & - & - & - & & - \\
\hline 8 & $T^{3}$ & - & - & - & - & - & & - \\
\hline
\end{tabular}

\section{CONCLUSIONES}

4.1 Los rotíferos en estanques de tierra aparecieron al segundo día de iniciado el ensayo.

4.2 La dosis de los fertilizantes utilizados: gallinaza más superfosfato, que permitió la producción masiva de rotíferos del género Brachionus, oscilé entre 1 a $1.5 \mathrm{~kg} / \mathrm{m} 2$ de gallinaza, más $30 \mathrm{~g}$. De superfosto/m2; el empleo de cantidades menores o mayores a este rango, hizo que la producción de rotíferos sea mínima 0 ausente.

4.3 La producción máxima de rotíferos del género Brachionus fue de 78 × 103 $\mathrm{ml} / \mathrm{l}$ y se obtuvo al quinto día, en el estanque $\mathrm{N} \cong 6$. 


\section{BIBLIOGRAFIA}

AMAT, F. 1987. Cultivos de Especies Auxiliares en Acuicultura. Curso Internacional del 23-28 marzo, Huacho - Perú. 28 pp.

FERNANDEZ, A. 1982. Guía para el Estudio de Algas. Departamento de Ciencias Biológicas, Universidad Nacional de Trujillo, 211 Pp.

KOSSOWSKI, C. 1980. Ensayo de Reproducción Inducida en Palometa "Carachica» M ylossomadutiventris (CUVIER) 1818 (Piscescypriniformes) con el uso de GCH. A cta Cientifica Venezolana 31 444-448.

OLIVIER, R. 1962. Rotíferos Plantónicos de Argentina, Universidad Nacional de la Plata, Facultad de Ciencias Naturales y M useo; Tomo VIII; Zoología, №63: $365 p$.

PENNAK, W. 1953. The freswater Invertebrates of the United States, Capítulo VIII; Rotifers. Editorial Ronald pares, $213 \mathrm{pp}$.

RODRIGUEZ, A. 1983. Cultivo masivo de Brachionus plicatilis O.F. Muller Rot!fero), alimentado con $\mathrm{N}$ annochloria badillaris, Planta de Microalgas (Sausal), Cap. Casa Grande L tda. 20p.

TECH, E. 1981. Cultura of Zooplancton (Brachionus and M oma) Report of the training couseon Crowing food organisms, forfishatcheries, Tigbadan, Iloilo, Philipines, 3-33 A ugust 225p. 35 - 51.

WOYNAROWICH, E. y L. HORVATH 1981. Propagación artificial de peces de aguas templadas. M anual para Extensionistas, FAO Doc. Tc. Pesca (201) 187 p. 101117.

VILLEGAS, C. 1981. Culture and screening of food organisms As potential larval food forfinfish and shellfish. Report of thetraining course on crowingfood organisms for fish hatcheries tigbauan, Iloilo, Philipines 3 -22 A ugust. 225p. 9 - 15. 